\title{
Anestesia locorregional en una paciente adulta con síndrome de Prader-Willi: consideraciones anestésicas
}

\author{
Anesthetic consideration: Regional anesthesia with \\ Prader-Willi
}

José Félix Martínez Juste', Jara Altemir Trallero', José Antonio Gimeno Orna

\begin{abstract}
Prader-Willi syndrome (PWS) is a disorder caused by a genetic alteration that causes a multisystem clinic. It can be due mainly to three genetic mechanisms; a paternal deletion of the 15q11-13 region, a maternal uniparental disomy, or an imprinting defect. The paternal deletion is observed in $70 \%$ of the patients, the disomy in $25 \%$ and the imprinting defect in only $5 \%$ of those affected by this syndrome. 1) It is the most common syndromic cause of obesity with an estimated prevalence in the population of $1: 50,000 ; 2$ ) The clinic is very variable, which is why clinical criteria have been created that, supported by the genetic study, confirm the diagnosis; 3) They have difficulty feeding during lactation, which leads to hyperphagia in childhood that leads to obesity. In the adult stage, in addition to obesity, respiratory pathology, sleep disturbances and psychological disorders stand out; 4) Objective: the aim of the present review was to compile the cases recorded in the scientific literature of patients anesthetized with PWS and the anesthetic options used in said patients.
\end{abstract}

\section{RESUMEN}

El síndrome de Prader-Willi (SPW) es un trastorno causado por una alteración genética que provoca una clínica multisistémica. Puede ser debido principalmente a tres mecanismos genéticos; una deleción paterna de la región 15q11-13, una disomía uniparental materna o un defecto de impronta. La deleción paterna se observa en el $70 \%$ de los pacientes, la disomía en el $25 \%$ y el defecto de impronta en tan solo el $5 \%$ de los afectados por este síndrome. 1) Constituye la

\section{Key words:}

Prader Willi syndrome, anesthetic considerations

\section{Palabras clave:}

Síndrome de Prader Willi, anestesia

Anestesiología y Reanimación, Hospital Clínico Universitario Lozano Blesa. Zaragoza, España.

Fecha de ingreso: 12 de septiembre de 2018

Fecha de aceptación: 22 de febrero de 2019

\section{ORCID}

https://orcid.org/0000-0003-3031-8450

Correspondencia:

José Félix Martínez Juste

Email: josemtezjuste@gmail.com 
causa sindrómica más frecuente de obesidad con una prevalencia estimada en la población de 1:50.000; 2) La clínica es muy variable por lo que se han creado unos criterios clínicos que apoyados por el estudio genético confirman el diagnóstico; 3) Presentan dificultad para la alimentación durante la lactancia, que da paso a una hiperfagia en la infancia que deriva en obesidad. En la etapa adulta, además de la obesidad destacan la patología respiratoria, alteraciones del sueño y trastornos psicológicos; 4) Objetivo: el objetivo de la presente revisión fue recopilar los casos registrados en la literatura científica de pacientes anestesiados con SPW y las opciones anestésicas utilizadas en dichos pacientes.

\section{Caso clínico}

P resentamos el caso de una paciente de 40 años diagnosticada con SPW y programada para la realización de fistulectomía perianal. El estudio preoperatorio presentaba como principales características: obesidad (IMC $=41$ ), fumadora de 30 cigarrillos al día, disfunción ventilatoria con componente restrictivo y obstructivo, síndrome de apnea hipopnea obstructiva del sueño (SAHOS) en tratamiento con BiPAP, trastorno de ansiedad generalizada, hipotiroidismo, hipertensión arterial y anemia ferropénica. Su tratamiento habitual era: formeterol y beclometasona inhalados, topiramato, eutirox, torasemida y hierro oral.

En los exámenes preoperatorios, el electrocardiograma y la radiografía de tórax eran normales. Las pruebas funcionales respiratorias corroboraron la presencia de patrón respiratorio mixto (FEV1/FVC: 71\%, FVC: 75\%, FEV1: 66\%).

Lo más destacable en su fenotipo era: obesidad troncular, estrabismo, epicanto y cifoescoliosis. En la valoración de la vía aérea se observó macroglosia, leve micrognatia, Mallampati clase III, adecuada movilidad cervical y distancia tiromentoniana mayor de $6,5 \mathrm{~cm}$.

Se premedicó a la paciente la noche anterior a la intervención con bromazepam y se tuvo especial cuidado en vigilarla durante el período nocturno para evitar la ingesta de alimentos respetando así el ayuno preoperatorio de seis horas. Además, una hora antes de la cirugía se completó la premedicación con el tratamiento broncodilatador inhalado, junto con ranitidina $50 \mathrm{mg}$ y metoclopramida $10 \mathrm{mg}$ endovenosos.

Se realizó una anestesia locorregional subaracnoídea asociándose sedación con $2 \mathrm{mg}$ de midazolam endovenosos. La punción se llevó a cabo con una aguja tipo Whitacre calibre $25 \mathrm{G}$ en el espacio interespinoso L3-L4 utilizando $5 \mathrm{mg}$ de bupivacaína hiperbárica $0,5 \%$ y 10 microgramos de fentanilo. Además de la monitorización básica habitual, se utilizó cánula nasal con capnógrafo.
El bloqueo anestésico de la zona perineal fue efectivo, completándose la fistulectomía sin incidencias. Durante el período intraoperatorio la paciente se mantuvo estable hemodinámicamente en todo momento y con una saturación de oxígeno de $95 \%$ con oxígeno suplementario a través de una cánula nasal a 3 litros por minuto. Durante el posoperatorio, la analgesia se realizó con $50 \mathrm{mg}$ de dexketoprofeno y $1 \mathrm{~g}$ de paracetamol endovenosos cada 8 horas, evitando el uso de opioides. La paciente fue dada de alta a las 24 horas de la intervención quirúrgica sin presentarse eventos de interés.

\section{Discusión}

El SPW constituye un reto para el anestesiólogo debido a sus peculiaridades clínicas y a la patología asociada al síndrome. Existen pocos casos publicados que se refieren a las consideraciones anestésicas en estos pacientes, siendo la mayoría de ellos casos realizados con anestesia general y en pacientes pediátricos.

Aunque el SPW ha sido ampliamente estudiado en los niños, se conoce mucho menos acerca de su evolución en la etapa adulta. La obesidad, el compromiso respiratorio y las enfermedades cardiovasculares asociadas al síndrome constituyen los principales problemas anestésicos en los pacientes adultos.

Una de las mayores preocupaciones anestésicas en los pacientes con SPW la constituye el manejo de la vía aérea. Algunos autores describen dificultades en la intubación endotraqueal en pacientes con este síndrome[5],[6]. En estos pacientes deberían estar previstas las medidas oportunas para afrontar la posibilidad de una dificultad en la ventilación e intubación. Además de la obesidad, existen otros aspectos propios del fenotipo de los pacientes con SPW que podrían contribuir a aumentar la dificultad para la intubación como la micrognatia, la macroglosia, las alteraciones 
en el paladar, los defectos en la dentición y la limitación en la movilidad del cuello.

En nuestro caso, debido a la localización de la zona quirúrgica y a la breve duración prevista de la intervención, se optó por una anestesia locorregional con el fin de evitar así el abordaje de la vía aérea en una paciente con alta probabilidad de presentar dificultad para la intubación endotraqueal (obesidad, macroglosia, micrognatia, Mallampati clase III).

Existe un riesgo aumentado de broncoaspiración en los pacientes con este síndrome, debido a la presencia de hipotonía, obesidad, saliva espesa, reflujo gastroesofágico, ventilación-intubación difícil y un cuadro de dilatación aguda gástrica (causado por la gran ingesta de alimentos asociada a la dificultad para vomitar y un umbral del dolor muy elevado)[3]; además, la ausencia de sensación de saciedad lleva a estos pacientes a una ingesta compulsiva de alimentos muchas veces de forma subrepticia, lo que dificulta el cumplimiento del tiempo adecuado de ayuno preoperatorio. Sloan y cols. sugieren considerar el alto riesgo de aspiración y tomar las medidas preventivas adecuadas en todos los pacientes con SPW[7]. En el caso que describimos, se vigiló estrechamente a la paciente para que cumpliera el ayuno de forma correcta y se premedicó con metoclopramida y ranitidina antes de la intervención.

Un evento relacionado con la vía aérea y descrito en varios casos es la presencia de un episodio de broncoespasmo tras la intubación o durante el intraoperatorio[6],[8],[9]. En nuestro caso, los antecedentes de tabaquismo y de bronquitis crónica de tipo mixto hacían prever una hiperreactividad de la vía aérea por lo que además de evitar la intubación con la anestesia regional se premedicó con su tratamiento inhalador habitual.

Los pacientes adultos con SPW son más propensos a presentar insuficiencia respiratoria e infecciones respiratorias durante el período posoperatorio que pueden verse agravadas en caso de asociarse a síndrome de apnea obstructiva del sueño. La hipotonía y la debilidad muscular provocan alteraciones respiratorias de tipo restrictivo o mixto y junto con la dificultad para expectorar de forma eficaz, facilitan las infecciones respiratorias que se dan casi en el $50 \%$ de los adultos con este síndrome[10]. Además, la presencia de obesidad y escoliosis en estos pacientes contribuye a empeorar la insuficiencia respiratoria restrictiva[11].

En el caso aquí descrito, la paciente presentaba el antecedente de disfunción ventilatoria con componente restrictivo y obstructivo asociada a SAHOS, además de una marcada escoliosis. Con la realización de una anestesia locorregional utilizando una baja dosis de anestésico local se intentó disminuir las probabilidades de insuficiencia o infección respiratoria en el posoperatorio al no afectarse la ventilación ni la capacidad para toser de la paciente. Tseng y cols. recomiendan la estancia en una Unidad de Cuidados Intensivos (UCI) para todos aquellos pacientes con SPW asociado a SAHOS, incluso cuando no se han utilizado derivados opiáceos ni relajantes musculares[9]. En nuestro caso, al no haberse realizado una anestesia general, decidimos evitar la estancia en $\mathrm{UCI}$ y mantener a la paciente en observación durante las primeras 24 horas continuando con su tratamiento con BiPAP.

La sedación en el SPW facilita la colaboración para realizar el bloqueo raquídeo y disminuye la ansiedad durante el trascurso de la cirugía. La hipotonía de estos pacientes los hace más sensibles a los fármacos sedantes, que deben administrarse con precaución, ya que pueden llegar a provocar depresión respiratoria. En nuestro caso, se administraron benzodiacepinas de vida media corta vía oral la noche anterior y vía endovenosa durante la cirugía. Y se utilizó cánula nasal con capnografía para detectar precozmente alguna apnea en caso de producirse.

Aunque en la mayoría de casos registrados de intervenciones quirúrgicas en pacientes con SPW se realizó una anestesia general, Legrand y cols., describieron una serie de cuatro casos, que incluía pacientes pediátricos en los que se utilizó anestesia locorregional como analgesia posoperatoria o como forma primaria de anestesia asociando sedación con ketamine[12].

\section{Conclusiones}

El SPW representa un reto para el anestesiólogo. La conducta anestésica debe prestar especial interés en el manejo de la vía aérea y tener en cuenta el riesgo de broncoaspiración y de complicaciones posoperatorias.

Con este caso proponemos la anestesia locorregional en adultos con SPW como una opción anestésica a tener en cuenta, que podría evitar las posibles complicaciones respiratorias y relacionadas con la vía aérea, pero son necesarias las publicaciones de más series de casos de pacientes adultos con SPW sometidos a anestesia locorregional para determinar la seguridad y eficacia de esta técnica. 


\section{Referencias}

1. Cassidy SB, Forsythe $M$, Heeger S, Nicholls RD, Schork N, Benn P, et al. Comparison of phenotype between patients with PraderWilli syndrome due to deletion $15 q$ and uniparental disomy 15 . Am J Med Genet. 1997;68:433440.11

2. Butler MG. Prader-Willi syndrome: current understanding of cause and diagnosis. Am J Med Genet. 1990 Mar;35(3):319-32. https://doi. org/10.1002/ajmg.1320350306 PMID:2309779

3. Gunay-Aygun M, Schwartz S, Heeger S, O'Riordan MA, Cassidy SB. The changing purpose of Prader-Willi syndrome clinical diagnostic criteria and proposed revised criteria. Pediatrics. 2001 Nov; 108(5):E92. https://doi. org/10.1542/peds.108.5.e92 PMID:11694676

4. Solá-Aznar J, Giménez-Pérez G. Abordaje integral del síndrome de Prader-Willi en la edad adulta. Endocrinol Nutr. 2006;53(3):181-
9. https://doi.org/10.1016/ S1575-0922(06)71086-5.

5. Mayhew JF, Taylor B. Anaesthetic considerations in the PraderWilli syndrome. Can Anaesth Soc J. 1983 Sep;30(5):565-6. https:// doi.org/10.1007/BF03007103 PMID:6138138

6. Lirk P, Keller C, Colvin J, Rieder J, Wulf K. Anaesthetic management of the Prader-Willi syndrome. Eur J Anaesthesiol. 2004 Oct;21(10):831-3. PMID:15678742

7. Sloan TB, Kaye Cl. Rumination risk of aspiration of gastric contents in the Prader-Willi syndrome. Anesth Analg. 1991 Oct;73(4):492-5. https://doi. org/10.1213/00000539199110000-00023 PMID:1897775

8. Kawahito $\mathrm{S}$, Kitahata $\mathrm{H}$, Kimura $\mathrm{H}$, Kohyama A. [Bronchospasm during anesthesia in a patient with Prader-Willi syndrome]. Masui. 1995 Dec;44(12):1675-9. PMID:8583665

9. Tseng $\mathrm{CH}$, Chen $\mathrm{C}$, Wong $\mathrm{CH}$, Wong SY, Wong KM. Anesthe- sia for pediatric patients with Prader-Willi syndrome: report of two cases. Chang Gung Med J. 2003 Jun;26(6):453-7. PMID:12956294

10. Butler JV, Whittington JE, Holland AJ, Boer H, Clarke D, Webb T. Prevalence of, and risk factors for, physical ill-health in people with Prader-Willi syndrome: a population-based study. Dev Med Child Neurol. 2002 Apr;44(4):248-55. https://doi.org/10.1017/ S001216220100202X PMID:11995893

11. Nixon GM, Brouillette RT. Sleep and breathing in Prader-Willi syndrome. Pediatr Pulmonol. 2002 Sep;34(3):209-17. https:// doi.org/10.1002/ppul.10152 PMID:12203850

12. Legrand R, Tobias JD. Anesthesia and Prader-Willi syndrome: preliminary experience with regional anesthesia. Paediatr Anaesth. 2006 Jul; 16(7):71222. https://doi.org/10.1111/ j.1460-9592.2006.01968.x PMID:16879513 